\title{
Article
}

\section{Effects of Cardiac Structural Remodelling During Heart Failure on Cardiac Excitation - Insights from a Heterogeneous 3D Model of the Rabbit Atria}

Kottas, Petros, Colman, Michael A, Stephenson, Robert S, Castro, Simon J, Hart, George, Jarvis, Jonathan C, Boyett, Mark and Zhang, Henggui

Available at http://clok.uclan.ac.uk/13942/

Kottas, Petros, Colman, Michael A, Stephenson, Robert S, Castro, Simon J, Hart, George, Jarvis, Jonathan C, Boyett, Mark and Zhang, Henggui (2015) Effects of Cardiac Structural Remodelling During Heart Failure on Cardiac Excitation - Insights from a Heterogeneous 3D Model of the Rabbit Atria. Computing in Cardiology, 42 . pp. 969-972. ISSN 2325-8861

It is advisable to refer to the publisher's version if you intend to cite from the work.

For more information about UCLan's research in this area go to http://www.uclan.ac.uk/researchgroups/ and search for <name of research Group>.

For information about Research generally at UCLan please go to http://www.uclan.ac.uk/research/

All outputs in CLoK are protected by Intellectual Property Rights law, including Copyright law. Copyright, IPR and Moral Rights for the works on this site are retained by the individual authors and/or other copyright owners. Terms and conditions for use of this material are defined in the policies page. 


\title{
Effects of Cardiac Structural Remodelling During Heart Failure on Cardiac Excitation - Insights from a Heterogeneous 3D Model of the Rabbit Atria
}

\author{
Petros Kottas ${ }^{1}$, Michael A. Colman ${ }^{1}$, Robert S. Stephenson ${ }^{2}$, Simon J. Castro ${ }^{1}$, George Hart ${ }^{1}$, \\ Jonathan C. Jarvis ${ }^{2}$, Mark Boyett ${ }^{1}$, Henggui Zhang ${ }^{1}$ \\ ${ }^{1}$ University of Manchester, Manchester, UK \\ ${ }^{2}$ Liverpool John Moores University, Liverpool, UK
}

\begin{abstract}
Heart failure is a leading cause of morbidity and mortality in the western world. One of the effects of heart failure is the structural remodelling of cardiac tissue, including tissue dilation and development of fibrosis. It is therefore important to study these changes and their effect on cardiac activity, in order to gain a better understanding of the underlying mechanisms in arrhythmogenesis, which will hopefully enable us to develop better treatments for heart failure.

In this study we developed biophysically detailed models of the rabbit atria for normal and heart failure conditions. These models were used to study the effects of structural remodelling of heart failure on cardiac excitation wave conduction. Anatomical reconstructions of the control and heart failure hearts were based on contrast enhanced micro-CT imaging. Fibre orientation was extracted from the control and heart failure datasets. Effects of heart failure geometry on the activation pattern of atrial excitation waves were analyzed.

It was found that atrial activation time increased from the control to the heart failure case in both isotropic and anisotropic conditions, which is attributed primarily to the dilation of tissue caused by heart failure.
\end{abstract}

\section{Introduction}

Clinically, heart failure (HF) is the inability of the heart to pump blood sufficiently in order to meet the body's needs. Causes of HF include coronary artery disease, atrial fibrillation (AF) and myocardial infarction. It is associated with increased risks of cardiac arrhythmias in the atria, as there is a high prevalence of $\mathrm{AF}$ in patients with HF, and is forming a leading cause of morbidity and mortality in the western world [1]. Clinical and animal model studies have shown that HF is associated with cardiac structural remodelling, which includes cellular hypertrophy, tissue dilation, wall thinning and disruption of tissue fibre orientation $[2,3,4]$. However, the functional impact of such structural remodelling on cardiac excitation wave conduction in the atria and therefore its potential role in arrhythmogenesis is unclear. It is hypothesised that the structural changes in the atrial tissue during $\mathrm{HF}$ provide a substrate for the development of $\mathrm{AF}$. The aim of this study is to investigate HF-induced structural remodelling on atrial excitation wave conduction.

\section{Methods}

To study the effects of structural remodelling in the atrium, multi-scale electrophysiologically detailed computer models of the rabbit atria were developed. High resolution micro CT datasets were made by $\mathrm{Dr}$ Stephenson and Professor Jarvis as previously described, using hearts taken from an experimental series run in Liverpool and Manchester in collaboration with Professor G Hart [5,6]. Using image-based analysis, the model was segmented into distinct regions of the atria and tissue fibre orientation was extracted. Simulations were conducted in both anisotropic and isotropic conditions to assess the effect of fibre orientation on atrial activation in normal and HF conditions.

\subsection{Anatomical Reconstructions of the Rabbit Atria}

The 3D model of the atria was based on anatomical datasets of the rabbit atria, reconstructed from contrast enhanced micro-CT imaging in control and experimental HF. The HF dataset was obtained from a rabbit heart which was subjected to aortic valve destruction followed by aortic banding [5]. The datasets were obtained at a spatial resolution of $20 \mu \mathrm{m}$, which were downsampled to a resolution of $120 \mu \mathrm{m}$ in order to reduce computation time of the model simulations.

Based on prior knowledge of atrial anatomy and 

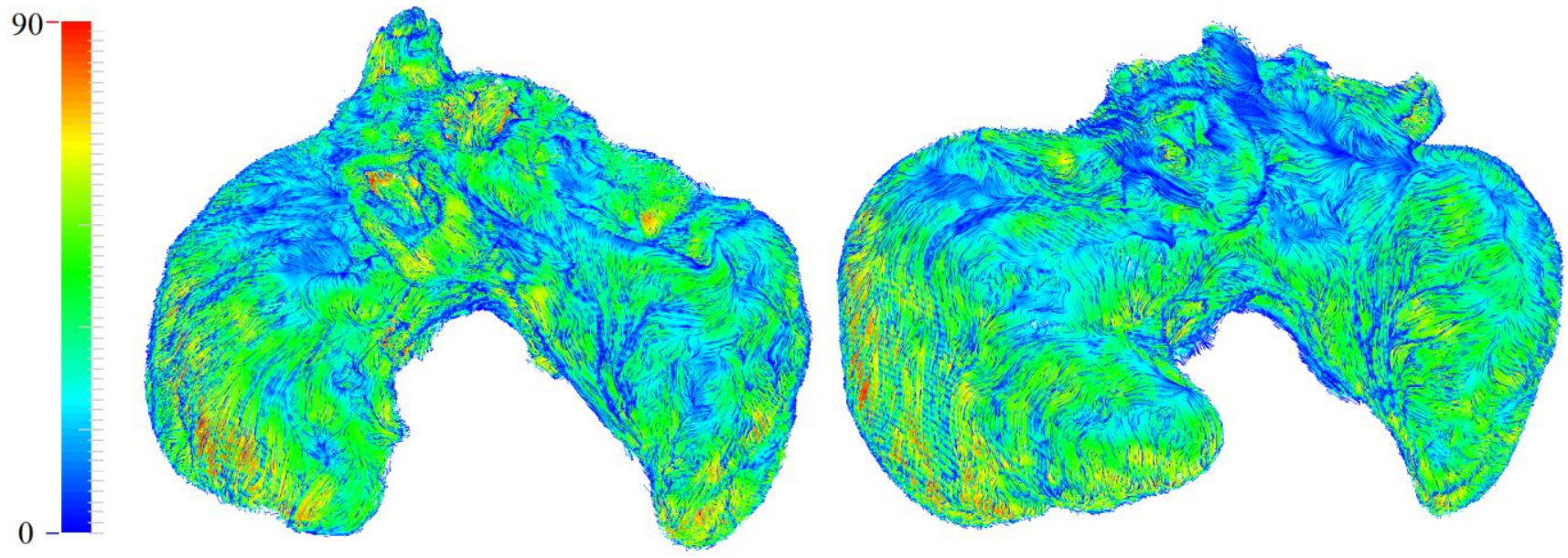

Figure 1: Fibre orientation visualization in the control (left) and HF (right) case. The view in both hearts is from the anterior - superior direction. Colouring represents fibre angle compared to the vertical axis.

electrophysiology, a conduction block zone region as observed in the rabbit heart was also simulated between the sinoatrial node and the atrial septum in both control and HF geometries. The existence and location of the block zone was validated against experimental data to ensure that the activation pattern agrees with experimental data.

Conduction velocity throughout the atrial tissue in both control and HF was adjusted through the diffusion coefficient in each case according to experimental data from rabbit as shown in Table 1.

\begin{tabular}{cc}
\hline & $\begin{array}{c}\text { Conduction Velocity } \\
(\mathrm{cm} / \mathrm{s})\end{array}$ \\
\hline Along the fibres (axial) & 70 \\
Across the fibres & 50 \\
(transverse) & 60 \\
Without fibres &
\end{tabular}

Table 1: Conduction velocity values within the atrial tissue.

\subsection{Fibre Orientation}

Fibre orientation was extracted from the high resolution contrast enhanced micro-CT imaging datasets in both control and HF cases by using intensity based structure tensor analysis [7]. A visualization of the extracted fibre directions is presented in Figure 1.

\subsection{Tissue Modelling}

To simulate the propagation through the rest of the atrial tissue, the Right Atrium rabbit model [8] was used. The action potential morphology and characteristics are shown in Figure 2.

Electrical excitation throughout the tissue is described by the following differential equation:

$$
\frac{\partial V}{\partial t}=\nabla \cdot D \nabla V-\frac{I_{i n}}{C_{m}}
$$

Where $\mathrm{V}$ is membrane potential, $\mathrm{t}$ is time, $\mathrm{D}$ is the diffusion coefficient for the voltage spread across the tissue, $I_{i o n}$ is the total ionic current and $C_{m}$ is the membrane capacitance.

The above equation was solved using the Finite Difference Method with a space step of $120 \mu \mathrm{m}$ and time step of $2 \mu \mathrm{sec}$.

In both control and $\mathrm{HF}$ simulations, the respective geometries were segmented in two regions; the sinoatrial node region and atrial tissue. The sinoatrial node region served as an initiation point for the excitation wave and was paced manually by applying a stimulus every $300 \mathrm{~ms}$.

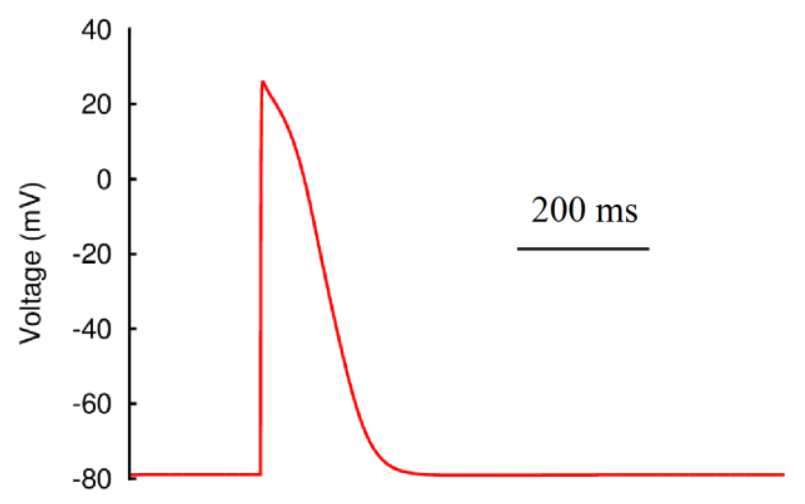

Figure 2: Action potential morphology of the rabbit right atrial single cell model. Action potential duration $\left(\mathrm{APD}_{90}\right)$ is $82 \mathrm{~ms}$ and amplitude is $105 \mathrm{mV}$. 
The simulations were run on a system running Scientific Linux with two Intel Xeon E5 2680v2 processors and $128 \mathrm{~GB}$ of RAM.

\section{Results}

Atrial activation time was longer in the $\mathrm{HF}$ case compared to the control case in both anisotropic (by 15\%) and isotropic (17\%) conditions, due to the increased tissue volume in HF (Figure 3). Consideration of fibre orientation in the models resulted in anisotropic conduction patterns with preferential conduction pathways in the atria. Remodelling of cell orientation in HF did not generate any additional slowing in atrial activation above the slowing calculated solely on the change in the geometry.

\subsection{Activation Pattern}

Activation throughout the rabbit atria in each case started from the sinoatrial node region where the stimulus was applied and continued to the right atrium, and towards the left atrium by propagating around the block zone, as seen experimentally. With the inclusion of fibres, activation was found to accelerate in regions where the

\section{A}
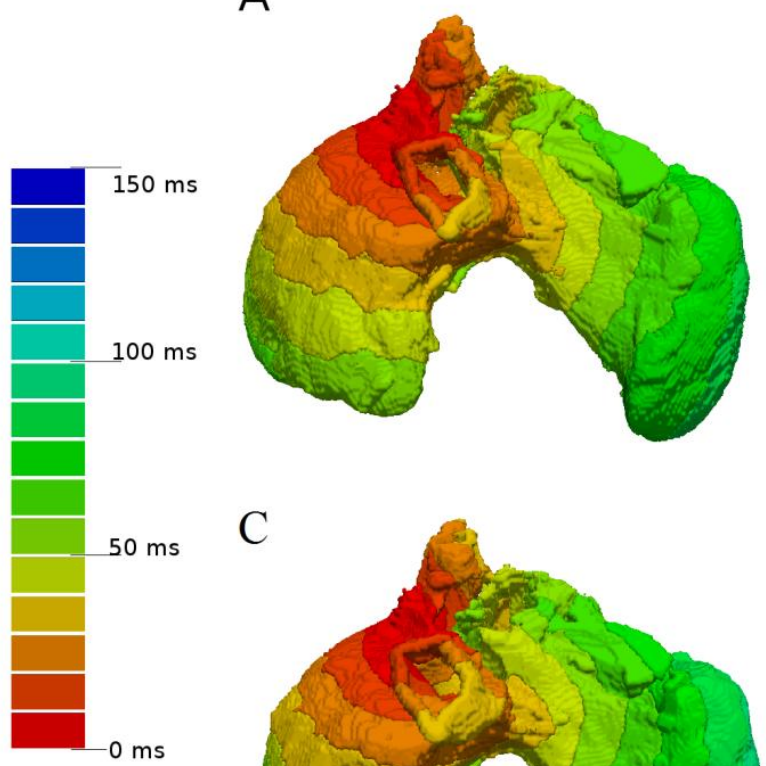

$\mathrm{C}$

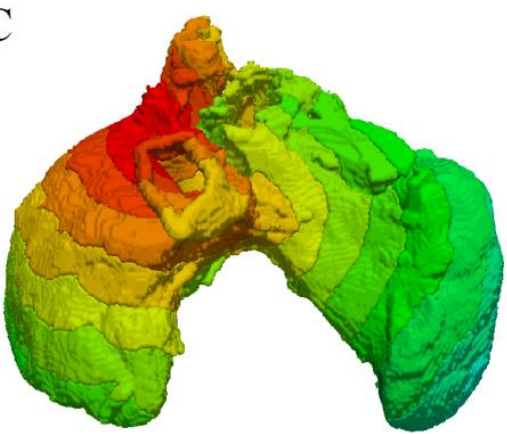

presence of aligned fibre bundles was more prominent.

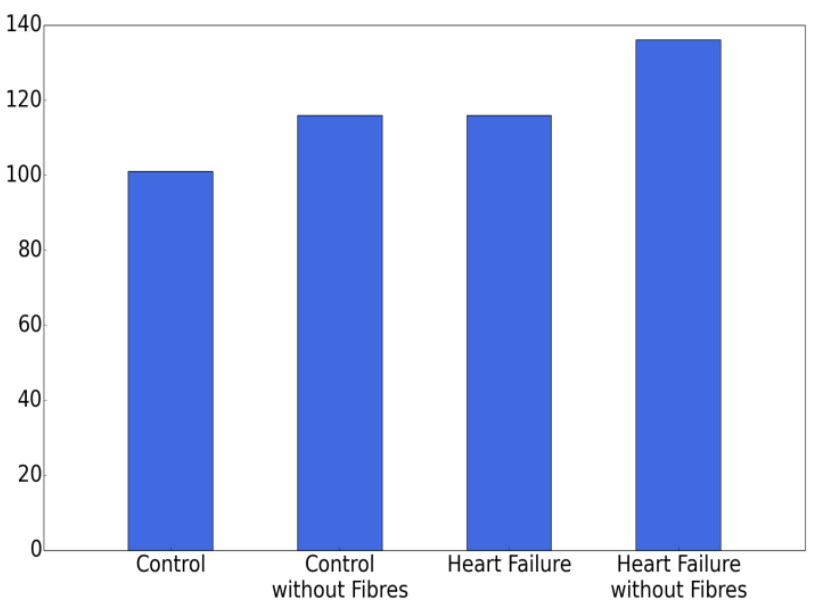

Figure 3: Total activation time of the atria in milliseconds in each case study.

Total activation time increased from $101 \mathrm{~ms}$ in the control case to $116 \mathrm{~ms}$ in the HF case when anisotropy is included in the model, as shown in Figure 4. More specifically, latest activation of the Right Atrium in the control case is at $63 \mathrm{~ms}$ while for the HF case at $74 \mathrm{~ms}$.

\section{B}

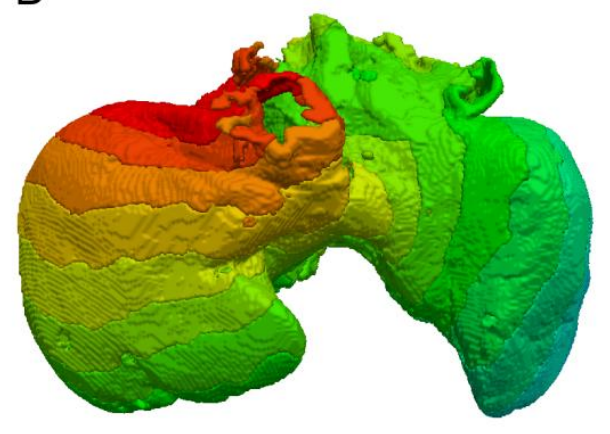

D

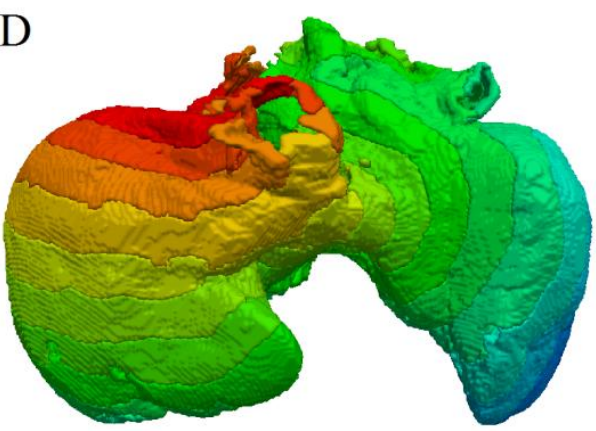

Figure 4: Activation Pattern in the Control (A), Heart Failure (B), Control without fibres (C) and Heart failure without fibres (D) case. Each colour represents a 10 millisecond advancement in activation time. 
Earliest activation of the LA in control is at $33 \mathrm{~ms}$ while in $\mathrm{HF}$ it is at $44 \mathrm{~ms}$. Finally, latest activation of the LA in the control case is at $101 \mathrm{~ms}$ and $116 \mathrm{~ms}$ in the HF case.

Similar changes occur in the isotropic case. Latest RA activation happens at $72 \mathrm{~ms}$ in control and $94 \mathrm{~ms}$ in $\mathrm{HF}$, while earliest LA activation is at $38 \mathrm{~ms}$ and $55 \mathrm{~ms}$ respectively. Finally, latest LA activation occurs at $116 \mathrm{~ms}$ in control and $136 \mathrm{~ms}$ in HF.

\subsection{Fibre Orientation}

Analysis of the fibre orientation data indicates that while there are preferred conduction pathways in the atria where fibre bundles are more likely to be observed, no significant disorganisation of fibres seems to be induced by $\mathrm{HF}$ in these regions.

An interesting consequence of the tissue dilation found in the HF case, mostly in the left and right atria and atrial appendages, is the increased radius of curvature of myocyte aggregates in those regions. Due to the increased tissue volume, the chains of myocytes do not need to change direction as rapidly as the control case in order to follow the curvature of geometry in the area. This effect can be seen in Figure 1.

\section{Conclusions}

Using a computational model of the rabbit atria with high resolution anatomical reconstructions of a control and $\mathrm{HF}$ rabbit heart and cellular orientation extracted from the datasets, it was possible to investigate the effects of structural remodelling on cardiac excitation in the atria during $\mathrm{HF}$.

In both isotropic and anisotropic cases, activation time increased in HF compared to the control case. This was attributed to the highly dilated tissue found in the left and right atria and atrial appendages in the HF case, rather than the altered orientation. Such structural remodelling may facilitate re-entrant excitation waves in the atria.

Fibre orientation studies reveal that the fibre bundles forming in the rabbit atria play an important role directing the excitation wave along preferred conduction pathways in both control and HF cases, though no additional change in fibre disorganisation is seen due to HF remodelling in these regions.

The remodelled anatomical structure through its increase in volume has an effect on the fibre organisation in regions where tissue dilation is found most predominantly. This effect is manifested though a smoothing out of fibre angles, since fibre bundles curve around larger tissue volumes compared to the sharper edges of the control atria.

Further studies need to be performed to investigate higher degrees of tissue heterogeneity and automatic pacing of the rabbit atrium in order to get a better understanding of the effects of structural remodelling in atrial depolarisation.

\section{Acknowledgements}

This work was funded by an EPSRC grant (EP / J00958X/1) and by a Fellowship to RSS from The Alder Hey Children's Hospital Trust.

\section{References}

[1] Nohria A, Lewis E, Stevenson L. Medical Management of Advanced Heart Failure. JAMA. 2002;287(5):628-640.

[2] Kaori Shinagawa, Yan-Fen Shi, Jean-Claude Tardif, TackKi Leung, Stanley Nattel. Dynamic Nature of Atrial Fibrillation Substrate During Development and Reversal of Heart Failure in Dogs. Circulation 2002 Jun 4;105(22):2672-8

[3] Danshi Li, Samir Fareh, Tack Ki Leung, Stanley Nattel. Promotion of Atrial Fibrillation by Heart Failure in Dogs Circulation. 1999; 100: 87-9.

[4] Stanley Nattel, Ange Maguy, Sabrina Le Bouter, Yung-Hsin Yeh. Arrhythmogenic Ion Channel Remodeling in the Heart: Heart Failure, Myocardial Infarction, and Atrial Fibrillation. Physiol Rev 2007 87: 425-456.

[5] Robert S. Stephenson, Mark R. Boyett, George Hart, Theodora Nikolaidou, Xue Cai, Antonio F. Corno, Nelson Alphonso, Nathan Jeffery, Jonathan C. Jarvis. Contrast Enhanced Micro-Computed Tomography Resolves the 3 Dimensional Morphology of the Cardiac Conduction System in Mammalian Hearts. PLoS ONE 7(4): 10.1371/journal.pone.0035299

[6] Jonathan C. Jarvis, Robert S. Stephenson. Studying the Microanatomy of the Heart in Three Dimensions: A Practical Update. Front Pediatr. 2013; 1: 26.

[7] Haibo Ni, Simon J. Castro, Robert S Stephenson, Jonathan C Jarvis, Tristan Lowe, George Hart, Mark R Boyett, Henggui Zhang Extracting Myofibre Orientation from Micro-CT Images: An Optimization Study. Computing in Cardiology 2013; 40:823-826

[8] OV Aslanidi, RS Dewey, AR Morgan, MR Boyett, H Zhang. Electrophysiologically Detailed Models of the Right and Left Rabbit Atria: Pharmacological Impacts on Propagation and Arrhythmogenesis.. Computers in Cardiology 2008; 35:69-72

Address for correspondence:

Petros Kottas

Room 3.17

$3^{\text {rd }}$ Floor, Schuster Laboratory

Biological Physics Group,

School of Physics and Astronomy,

University of Manchester,

Oxford Road, Manchester, UK

M13 9PL,

Petros.Kottas@postgrad.manchester.ac.uk 